ФИБРИЛЛЯЦИЯ ПРЕДСЕРДИЙ И ФАКТОРЫ РИСКА ИНСУЛЬТА У ПАЦИЕНТОВ

С ИМПЛАНТИРОВАННЫМИ СЕРДЕЧНЫМИ УСТРОЙСТВАМИ

И.Р.Рахимова ${ }^{1}$, Т.Н.Хайбуллин ${ }^{1}$, В.В.Ковальчук ${ }^{2}$, А.С.Абдрахманов ${ }^{3}$

${ }^{1}$ НАО Медицинский университет Семей, Республика Казахстан, г. Семей, ул. Абая, д. 103,

${ }^{2}$ Кембриджский Университет, Великобритания, Кембридж СВ2 1TN, переулок Тринити, ${ }^{3}$ Национальный научный кардиохирургический центр, Республика Казахстан, г. Нур-Султан, пр. Туран, д. 38.

Цель. Определить долю и бремя вновь возникшей фибрилляции предсердий (ФП) у пациентов с имплантированными сердечными устройствами и без предшествующей ФП, а также оценить частоту встречаемости факторов риска инсульта у пациентов с ФП.

Методы исследования. Были проанализированы истории болезни 111 пациентов с имплантированными сердечными устройствами с функцией удаленного мониторинга. ФП, диагностированная устройством, была интерпретирована врачом-аритмологом и отобраны случаи достоверной ФП. Было проведено сопоставление группы пациентов с ФП и без нее по ряду факторов. С целью уточнения влияния факторов риска на продолжительность ФП, все случаи ФП были разделены на 3 категории в зависимости от бремени ФП за сутки. В первую группу вошли пациенты с бременем ФП за сутки $0,1 \%$ и менее $(n=10,(45,5 \%))$, во вторую - от 0,3\% до 12,2\% (n=7, $(31,8 \%))$, и в третью группу со $100 \%(\mathrm{n}=5,(22,7 \%))$. Пациенты с бременем 0,2\% и 12,3-99,9\% отсутствовали.

Результаты. Впервые выявленная ФП была зарегистрирована в 19,8\% случаев. Риск инсульта среди этих пациентов был высоким - 2 [1; 3] балла по шкале $\mathrm{CHA}_{2} \mathrm{DS}_{2}-\mathrm{VASc}$ у мужчин, и 3 [2,75; 3,75] балла у женщин. Артериальная гипертензия (АГ) 2 и 3 степени была зарегистрирована у всех пациентов в группе с бременем ФП $100 \%$ за сутки. Что статистически значимо отличалось от 2 других групп ( $=0,043)$. В группе с бременем ФП $100 \%$ уровни NT-proBNP, D-димера и креатинина были статистически значимо выше, чем в остальных группах $(\mathrm{p}=0,037, \mathrm{p}=0,031$ и $\mathrm{p}=0,036$ соответственно). При анализе зависимости уровня креатинина от наличия ФП площадь под ROC-кривой составила 0,653 [95\% ДИ: 0,528-0,779] (p=0,017). Частота стимуляции правого желудочка у пациентов с ИКД была выше в группе пациентов с ФП.

Заключение. ФП встречается у 1/5 пациентов с имплантированными сердечными устройствами. Все пациенты с ФП являлись потенциальными кандидатами на терапию антикоагулянтами из-за высокого риска инсульта. Суточное бремя ФП положительно коррелирует с наличием и степенью АГ, а также с маркерами дисфункции почек, хронической сердечной недостаточности и тромбоза. Повышенный уровень креатинина является предиктором развития ФП.

Ключевые слова: фибрилляция предсердий; риск инсульта; предикторы фибрилляции предсердий; имплантированные сердечные устройства; кардиовертер-дефибриллятор; сердечная ресинхронизирующая терапия

Конфликт интересов: авторы заявили об отсутствии потенциального конфликта интересов Рукопись получена: 07.06.2021 Исправленная версия получена: 19.09.2021 Принята к публикации: 20.09 .2021 Ответственный за переписку: Рахимова Идалия Рафаиловна, E-mail: idalya111@mail.ru

И.Р.Рахимова - ORCID ID 0000-0002-9011-2879, Т.Н.Хайбуллин - ORCID ID 0000-0003-1886-0538, В.В.Ковальчук ORCID ID 0000-0002-1004-2162, А.С.Абдрахманов - ORCID ID 0000-0001-6315-5016

Для цитирования: Рахимова ИР, Хайбуллин ТН, Ковальчук ВВ, Абдрахманов АС. Фибрилляция предсердий и факторы риска инсульта у пациентов с имплантированными сердечными устройствами. Вестник аритмологии. 2021;28(3): 28-36. https://doi.org/10.35336/VA-2021-3-28-36.

\title{
ATRIAL FIBRILLATION AND STROKE RISK FACTORS IN PATIENTS WITH CARDIAC IMPLANTABLE ELECTRONIC DEVICES \\ I.R.Rakhimova ${ }^{1}$, T.N.Khaibullin ${ }^{1}$, V.V.Kovalchuk ${ }^{2}$, A.S.Abdrakhmanov ${ }^{3}$ \\ ${ }^{1}$ NJSC Semey Medical University, Kazakhstan, Semey, 103 Abaya str., ${ }^{2}$ University of Cambridge, Great Britain, Cam- bridge CB2 ITN, Trinity Lane, ${ }^{3}$ National research cardiac surgery center, Kazakhstan, Nur-Sultan, 38 Turan avenue.
}

Purpose. Determination the proportion and burden of new-onset atrial fibrillation (AF) in patients with cardiac implantable electronic devices (CIED) and without prior AF and assessing the incidence of risk factors for stroke in patients with AF.

Methods. The medical history of 111 patients with CIED with remote monitoring function were analyzed. AF diagnosed by the device was interpreted by an arrhythmologist and cases of significant AF were selected. The group of patients with and without AF was compared for several factors. To clarify the influence of risk factors on the duration of 
$\mathrm{AF}$, all $\mathrm{AF}$ cases were divided into 3 categories depending on the AF burden per day. The first group included patients with an AF burden per day of $0.1 \%$ or less $(\mathrm{n}=10,(45.5 \%))$, the second - from $0.3 \%$ to $12.2 \%(\mathrm{n}=7,(31.8 \%))$, and the third group - with $100 \%(\mathrm{n}=5,(22.7 \%))$. Patients with a burden of $0.2 \%, 12.3-99.9 \%$ were absent.

Results. Newly diagnosed AF was registered in $19.8 \%$ of cases. The risk of stroke among these patients was high - $2[1 ; 3]$ points on the $\mathrm{CHA}_{2} \mathrm{DS}_{2}-\mathrm{VASc}$ for men, and $3[2,75 ; 3,75]$ points for women. Hypertension of 2 and 3 degrees was recorded in all patients in the group with an AF burden of $100 \%$ per day. That significantly differed from the other 2 groups ( $\mathrm{p}=0.043$ ). In the group with an AF burden of $100 \%$, the levels of NT-proBNP, D-dimer, and creatinine were significantly higher than in the other groups $(\mathrm{p}=0.037, \mathrm{p}=0.031$ and $\mathrm{p}=0.036$, respectively). When analyzing the dependence of creatinine level on the presence of AF, the area under the ROC-curve was 0.653 with $95 \%$ confidence interval $0.528-0.779(\mathrm{p}=0.017)$. The proportion of right ventricular pacing in patients with ICD was higher in the group of patients with AF.

Conclusion. AF occurs in $1 / 5$ of patients with CIED. All patients with AF were potential candidates for anticoagulation due to their high risk of stroke. The daily burden of AF is positively correlated with the presence and degree of hypertension, as well as with markers of renal dysfunction, chronic heart failure, and thrombosis. Elevated creatinine levels are a predictor of AF.

Keywords: atrial fibrillation; risk of stroke; predictors of atrial fibrillation; cardiac implantable electronic devices; cardioverter defibrillator; cardiac resynchronization therapy

Conflicts of Interests: nothing to declare

Received: 07.06.2021 Revision Received: 19.09.2021 Accepted: 20.09.2021

Corresponding author: Rakhimova Idaliya, E-mail: idalya111@mail.ru

I.R.Rakhimova - ORCID ID 0000-0002-9011-2879, T.N.Khaibullin - ORCID ID 0000-0003-1886-0538, V.V.Kovalchuk ORCID ID 0000-0002-1004-2162, A.S.Abdrakhmanov - ORCID ID 0000-0001-6315-5016

For citation: Rakhimova IR, Khaibullin TN, Kovalchuk VV, Abdrakhmanov AS. Atrial fibrillation and stroke risk factors in patients with cardiac implantable electronic devices. Journal of Arrhythmology. 2021;28(3): 28-36. https:// doi.org/10.35336/VA-2021-3-28-36.

Инсульт является грозным осложнением фибрилляции предсердий (ФП), которое возможно избежать. Субклиническая ФП, являющаяся находкой при анализе записей имплантированных сердечных устройств, имеет такие же последствия, как и клиническая ФП. Своевременное назначение антикоагулянтных препаратов, после оценки риска инсульта позволяет предотвратить это состояние [1-3]. ФП это суправентрикулярная тахиаритмия при которой наблюдается некоординированная электрическая активация предсердий и, как следствие, неэффективное сокращение предсердий. Клинические рекомендации указывают на необходимость записи ЭКГ, подтверждающей наличие ФП при помощи либо 12-канальной записи, либо одноканальной записи в течение не менее 30 секунд. Это является необходимым условием для рассмотрения вопроса о назначении антикоагулянтной терапии [4].

Целесообразность назначения и выбор препарата определяют врач совместно с пациентом в индивидуальном порядке. Согласно классификации, выделяют постоянные и непостоянные формы ФП. В случае, когда ФП имеет постоянный характер, не представляет трудности её зарегистрировать при помощи рутинной 12-канальный ЭКГ. Регистрация же пароксизмальной или персистирующей формы ФП представляет некоторые сложности, ввиду возможности ее бессимптомного или малосимптомного течения и кратковременного характера [3].

В данной ситуации на помощь приходят имплантированные сердечные устройства с предсердными электродами, позволяющие длительно мониторировать ритм предсердий. К имплантируемым сердечным устройствам относятся: постоянные электрокардиостимуляторы, имлантируемые кардиовертер-дефибрилляторы (ИКД) и сердечные устройства с ресинхронизирующей функцией (СРТ).

ИКД зарекомендовали себя в качестве инструмента профилактики у пациентов с риском внезапной сердечной смерти. Благодаря значительным технологическим достижениям имплантация ИКД стала проще и безопаснее. Благодаря улучшенным терапевтическим и диагностическим функциям устройства удалось добиться снижения смертности [5].

СРТ является стандартной терапией для пациентов с сердечной недостаточностью с нарушениями внутрижелудочковой проводимости [6]. Обнаружение у пациентов ФП, позволит провести более эффективную профилактику тромбоэмболических осложнений путем назначения антикоагулянтной терапии [7].

В современной научной литературе существует ряд работ, описывающих возникновение ФП у пациентов с имплантированными сердечными устройствами, а также факторы риска инсульта у данной когорты пациентов [8-10]. Однако, неясным остается вопрос частоты встречаемости впервые выявленной ФП у пациентов с ИКД и СРТ, так как данные литературы достаточно гетерогенны.

Цель исследования - оценить суточное бремя фибрилляции предсердий у пациентов с имплантированными сердечными устройствами, а также оценить частоту встречаемости факторов, связанных с возник- 
новением этого нарушения ритма сердца, и факторов риска инсульта.

\section{МАТЕРИАЛ И МЕТОДЫ ИССЛЕДОВАНИЯ}

Нами были проанализированы 114 историй болезней пациентов с имплантированными сердечными устройствами. Клинико-демографические и лабораторные данные получены из историй болезни. Критериями включения в исследование были: наличие имплантированных сердечных устройств, подключенных к системе удаленного мониторинга (Biotronik - Home Monitoring и Medtronic - CareLink) и отсутствие ФП в анамнезе и на момент имплантации устройства.

Система удаленного мониторинга активируется врачом после имплантации устройства, что позволяет с определенной периодичностью получать данные о работе устройства, записи эндограмм, а также «тревожные сообщения» при регистрации жизнеугрожающих аритмий $[11,12]$. Три истории болезни были исключены из анализа, ввиду неполных данных. Ста пациентам сердечные устройства были имплантированы в Национальном Научном Кардиохирургическом Центре г. Нур-Султан, остальным 11 пациентам в Больнице Скорой Медицинской Помощи г. Семей. Длительность исследования составила 8 лет (с 2013 по 2021 год). Исследование было одобрено локальным этическим комитетом.

Каждый эпизод, зарегистрированный устройством как ФП был оценен врачом-аритмологом и были отобраны истинные случаи ФП. В наше исследование вошли эпизоды с длительностью не менее 30 секунд.

Были собраны следующие клинико-демографические данные: пол, возраст, основные, сопутствующие, фоновые заболевания и осложнения, даты имплантации, тип имплантируемого устройства, лабораторные данные. У каждого пациента проведена оценка риска инсульта по шкале $\mathrm{CHA}_{2} \mathrm{DS}_{2}$-VASc. Количество баллов по шкале риска инсульта оцениваемое как высокое и требующее антикоагулянтной терапии было 2 у мужчин, и 3 у женщин [2].

Бременем ФП обозначается процентное соотношение времени, в течение которого у пациента регистрируется ФП во время наблюдения. В нашем исследовании мы оценивали бремя ФП за сутки. Бремя ФП за сутки у пациентов с выявленной ФП существенно различалось, и находилось в диапазоне от $0,1 \%$ до $100 \%$ за сутки. С целью уточнения взаимосвязи продолжительности ФП и ряда факторов, таких как пол, возраст, риск инсульта, сопутствующие заболевания, лабораторные данные, нами было принято решение разделить пациентов с ФП на 3 группы. В первую группу вошли пациенты с бременем ФП за сутки $0,1 \%$ и менее, во вторую - от 0,3\% до 12,2\%, и в третью группу со $100 \%$. Пациенты с бременем 0,2\%, и 12,3-99,9\% не были зарегистрированы в нашем исследовании.

\section{Статистическая обработка}

Статистический анализ проводился с использованием программы StatTech v. 1.2.0 (разработчик ООО “Статтех”, Россия). Количественные показатели оценивались на предмет соответствия нормальному распределению с помощью критерия Шапиро-Уилка (при числе исследуемых менее 50) или критерия Колмогорова-Смирнова (при числе исследуемых более 50). Количественные показатели, имеющие нормальное распределение, описывались с помощью средних арифметических величин (М) и стандартных отклонений (SD), границ 95\% доверительного интервала (95\% ДИ). В случае отсутствия нормального распределения

таблица 1.

\section{Характеристика пациентов (категориальные переменные)}

\begin{tabular}{|l|c|}
\hline Показатель & Значение \\
\hline Фибрилляция предсердий, n (\%) & $22(19,8)$ \\
\hline Мужчины, n (\%) & $82(73,9)$ \\
\hline Ишемическая КМП, n (\%) & $29(26,1)$ \\
\hline Дилятационная КМП, n (\%) & $13(11,7)$ \\
\hline Гипертрофическая КМП, n (\%) & $11(9,9)$ \\
\hline Постинфарктный кардиосклероз, n (\%) & $54(48,6)$ \\
\hline Инсульт в анамнезе, n (\%) & $10(9,0)$ \\
\hline Хроническая СН, n (\%) & $97(87,4)$ \\
\hline Сахарный диабет, n (\%) & $19(17,1)$ \\
\hline Дисфункция синусового узла, n (\%) & $3(2,7)$ \\
\hline Артериальная гипертензия, n (\%) & $53(47,7)$ \\
\hline Полная блокада ЛНПГ, n (\%) & $44(39,6)$ \\
\hline Приобретенный порок сердца, n (\%) & $4(3,6)$ \\
\hline Желудочковая тахикардия, n (\%) & $24(21,6)$ \\
\hline Кардиовертер-дефибриллятор, n (\%) & $60(54,1)$ \\
\hline СРТ-Д, п (\%) & $51(45,9)$ \\
\hline
\end{tabular}

Примечание: здесь и далее КМП - кардиомиопатия, $\mathrm{CH}$ - сердечная недостаточность, ЛНПГ - левая ножка пучка Гиса, СРТ-Д - сердечная ресинхронизирующая терапия с функцией дефибрилляции.

Таблица 2.

\section{Характеристика пациентов (количественные переменные)}

\begin{tabular}{|c|c|c|}
\hline Показатель & Значение & $95 \%$ ДИ \\
\hline Возраст, лет & 57 & $50-63,5$ \\
\hline ФВ ЛЖ, \% & 29 & $26-35$ \\
\hline Диаметр ЛП, см & 4,5 & $4,2-4,8$ \\
\hline $\mathrm{CHA}_{2} \mathrm{DS}_{2}$-VASc, баллов & 2 & $2-3,5$ \\
\hline 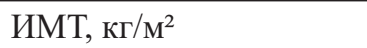 & $28,23 \pm 5,26$ & $27,17-29,3$ \\
\hline Длительность УМ, лет & 3,5 & $2-4,5$ \\
\hline NT-proBNP, пг/мЛ & 1876 & $1166-4474$ \\
\hline D-димер, мкг/мл & 0,37 & $0,23-1,19$ \\
\hline Креатинин, мкмоль/л & 88,4 & $75,78-106,1$ \\
\hline Глюкоза, мг/дл & 101,4 & $92,3-118,2$ \\
\hline Холестерин, ммоль/л & $4,85 \pm 1,32$ & $4,59-5,11$ \\
\hline Фибриноген, г/л & 3,3 & $2,7-3,93$ \\
\hline
\end{tabular}

Примечание: ФВ ЛЖ - фракция выброса левого желудочка, ЛП - левое предсердие, ИМТ - индекс массы тела, УМ - удаленный мониторинг. 
количественные данные описывались с помощью медианы $(\mathrm{Me})$ и нижнего и верхнего квартилей $\left(\mathrm{Q}_{1}-\mathrm{Q}_{3}\right)$.

Таблица 3.

Анализ клинических данных пациентов в зависимости от наличия ФП

\begin{tabular}{|c|c|c|c|c|}
\hline \multicolumn{2}{|l|}{ Показатель } & \begin{tabular}{|c|} 
Отсутствие \\
ФП
\end{tabular} & $\begin{array}{c}\text { Наличие } \\
\text { ФП }\end{array}$ & $\mathrm{p}$ \\
\hline \multirow{4}{*}{ КМП, n (\%) } & Отсутствие & $50(56,18)$ & $8(36,36)$ & \multirow{4}{*}{0,115} \\
\hline & ИКМП & $23(25,84)$ & $6(27,27)$ & \\
\hline & ДКМП & $10(11,24)$ & $3(13,64)$ & \\
\hline & ГКМП & $6(6,74)$ & $5(22,73)$ & \\
\hline \multirow{2}{*}{ ПИМ, n (\%) } & Отсутствие & $43(48,31)$ & $14(63,64)$ & \multirow{2}{*}{0,198} \\
\hline & Наличие & $46(51,69)$ & $8(36,36)$ & \\
\hline \multirow{2}{*}{$\begin{array}{l}\text { Инсульт } \\
\text { в анамнезе, n (\%) }\end{array}$} & Отсутствие & $80(89,89)$ & $21(95,45)$ & \multirow{2}{*}{0,683} \\
\hline & Наличие & $9(10,11)$ & $1(4,55)$ & \\
\hline \multirow{5}{*}{$\mathrm{XCH}, \mathrm{n}(\%)$} & Отсутствие & $11(12,36)$ & $3(13,64)$ & \multirow{5}{*}{0,063} \\
\hline & ХСН I ФК & $3(3,37)$ & $1(4,55)$ & \\
\hline & XCH II ФК & $9(10,11)$ & $7(31,82)$ & \\
\hline & XCH III ФК & $60(67,42)$ & $9(40,91)$ & \\
\hline & XCH IV ФК & $6(6,74)$ & $2(9,09)$ & \\
\hline \multirow{2}{*}{ СД, n (\%) } & Отсутствие & $74(83,15)$ & $18(81,82)$ & \multirow{2}{*}{1} \\
\hline & Наличие & $15(16,85)$ & $4(18,18)$ & \\
\hline \multirow{2}{*}{ ДСУ, n (\%) } & Отсутствие & $87(97,75)$ & \begin{tabular}{|l|}
$21(95,45)$ \\
\end{tabular} & \multirow{2}{*}{0,488} \\
\hline & Наличие & $2(2,25)$ & $1(4,55)$ & \\
\hline \multirow{4}{*}{$\begin{array}{l}\text { Степень АГ, } \\
\text { n (\%) }\end{array}$} & Отсутствие & $47(52,81)$ & $11(50)$ & \multirow{4}{*}{0,831} \\
\hline & АГ І степени & $4(4,49)$ & - & \\
\hline & АГ ІІ стенени & $13(14,61)$ & $3(13,64)$ & \\
\hline & АГ III степени & $25(28,09)$ & $8(36,36)$ & \\
\hline \multirow{2}{*}{$\begin{array}{l}\text { Наличие АГ, } \\
\text { n (\%) }\end{array}$} & Отсутствие & $47(52,81)$ & $11(50)$ & \multirow{2}{*}{0,813} \\
\hline & Наличие & $42(47,19)$ & $11(50)$ & \\
\hline \multirow{2}{*}{ ПБЛНПГ, n (\%) } & Отсутствие & $55(61,8)$ & $12(54,55)$ & \multirow{2}{*}{0,628} \\
\hline & Наличие & $34(38,2)$ & $10(45,45)$ & \\
\hline \multirow{4}{*}{ АВБ, n (\%) } & Отсутствие & $84(94,38)$ & $22(100)$ & \multirow{4}{*}{1} \\
\hline & АВБ І степени & $3(3,37)$ & - & \\
\hline & АВБ ІІ степени & $1(1,12)$ & - & \\
\hline & АВБ ІІІ степени & $1(1,12)$ & - & \\
\hline \multirow{2}{*}{ ППС, n (\%) } & Отсутствие & $87(97,75)$ & $20(90,91)$ & \multirow{2}{*}{0,175} \\
\hline & Наличие & $2(2,25)$ & $2(9,09)$ & \\
\hline \multirow{2}{*}{ ЖТ, n (\%) } & Отсутствие & $67(75,28)$ & $20(90,91)$ & \multirow{2}{*}{0,151} \\
\hline & Наличие & $22(24,72)$ & $2(9,09)$ & \\
\hline \multirow{2}{*}{ ХБП, n (\%) } & Отсутствие & $81(91,01)$ & $19(86,36)$ & \multirow{2}{*}{0,453} \\
\hline & Наличие & $8(8,99)$ & $3(13,64)$ & \\
\hline & 2 стадия & $2(66,7)$ & $1(33,3)$ & \\
\hline Стадия ХБП, & 3 стадия & $5(71,4)$ & $2(28,6)$ & 0,804 \\
\hline & 4 стадия & $1(100,0)$ & $0(0,0)$ & \\
\hline
\end{tabular}

Примечание: здесь и далее ИКМП, ГКМП и ДКМП - ишемическая, гипертрофическая и дилатационная КМП, ПИМ - перенесенный инфаркт миокарда, ХCH - хроническая СН, СД - сахарный диабет, ДСУ - дисфункция синусового узла, АВБ - атриовентрикулярная блокада, ППС приобретенный порок сердца, ХБП - хроническая болезнь почек.
Сравнение двух групп по количественному показателю, имеющему нормальное распределение, при условии равенства дисперсий выполнялось с помощью t-критерия Стьюдента. Сравнение двух групп по количественному показателю, распределение которого отличалось от нормального, выполнялось с помощью U-критерия Манна-Уитни. Категориальные данные описывались с указанием абсолютных значений и процентных долей. Сравнение процентных долей при анализе четырехпольных таблиц сопряженности выполнялось с помощью критерия хи-квадрат Пирсона (при значениях ожидаемого явления более 10), критерия Фишера (при значениях ожидаемого явления менее 10). Сравнение трех и более групп по количественному показателю, распределение которого отличалось от нормального, выполнялось с помощью критерия Краскела-Уоллиса, апостериорные сравнения - с помощью критерия Данна с поправкой Холма. Сравнение трех и более групп по количественному показателю, имеющему нормальное распределение, выполнялось с помощью однофакторного дисперсионного анализа, апостериорные сравнения проводились с помощью критерия Тьюки (при условии равенства дисперсий). Сравнение процентных долей при анализе многопольных таблиц сопряженности выполнялось с помощью точного критерия Фишера (при значениях ожидаемого явления менее 10). Апостериорные сравнения выполнялись с помощью критерия хи-квадрат Пирсона с поправкой Холма.

\section{ПОЛУЧЕННЫЕ РЕЗУЛЬТАТЫ}

Нами были проанализированы данные 111 пациентов с имплантированными сердечными устройствами, из которых 60 (54,1\%) - ИКД с первичной эндокардиальной системой, остальные 51 (45,9\%) СРТ. Средняя длительность наблюдения сердечного ритма при помощи опции удаленного мониторинга составила 3,5 года. Общая характеристика пациентов представлена в табл. 1 и 2.

\section{Анализ групп с выявленной ФП \\ и без нее}

Нами был проведен анализ ряда показателей в зависимости от наличия ФП. При анализе пола, возраста, индекса массы тела, риска инсульта по шкале $\mathrm{CHA}_{2} \mathrm{DS}_{2}$-VASc в зависимости от наличия ФП, не удалось установить статистически значимых различий. Доля пациентов с неадекватной антигипертензивной терапией в группе с ФП (n=8, (72,7\%)) была выше, чем в группе без ФП (n=18, $(42,9 \%))$. В 
группе пациентов с выявленной ФП (n=22) риск инсульта был оценен как высокий как среди мужчин, так и среди женщин. Для оценки риска кровотечений у данных пациентов была использована шкала HAS BLED. Средний балл был равен 2 [IQR 1;2]. Пациенты с баллом равным 3 (22,7\%) были расценены нами как пациенты с высоким риском кровотечения. Остальные 77,3 \% пациентов имели низкий риск кровотечения и, таким образом, не имели противопоказаний к терапии антикоагулянтами. Однако окончательное решение о приеме ОАК принималось в индивидуальном порядке с учетом множества факторов.

При анализе данных пациентов с ИКД нам удалось установить, что в группе с наличием ФП доля правожелудочковой стимуляции была статистически значимо выше (11\% Me [IQR 9,0; 53,5]), чем в группе без ФП (1\% Me [IQR 0,0; 5,5]) (p=0,043).

При проведении анализа диагноза пациента в зависимости от наличия ФП нам также не удалось выявить значимых различий. Нами был выполнен анализ лабораторных данных в зависимости от наличия ФП. Согласно полученным данным при оценке уровня креатинина в зависимости от наличия ФП, нами были установлены статистически значимые различия $(\mathrm{p}=0,027)$. При анализе других лабораторных данных в зависимости от наличия ФП, не удалось выявить статистически значимых различий. Результаты анализа представлены в табл. 3 и 4.

Оценка зависимости вероятности выявления ФП от уровня креатинина выполнялась с помощью ROC-анализа (рис. 1). Площадь под ROC-кривой составила 0,653 с 95\% ДИ: 0,528-0,779. Полученная модель была статистически значимой $(\mathrm{p}=0,017)$.

Пороговое значение уровня креатинина в точке cut-off, которому соответствовало наивысшее значение индекса Юдена, составило 103,4 мкмоль/л (рис. 2). При значении уровня креатинина выше или равном ему, прогнозировалось выявление ФП. Чувствительность и специфичность метода составили $54,5 \%$ и $76,7 \%$ соответственно.

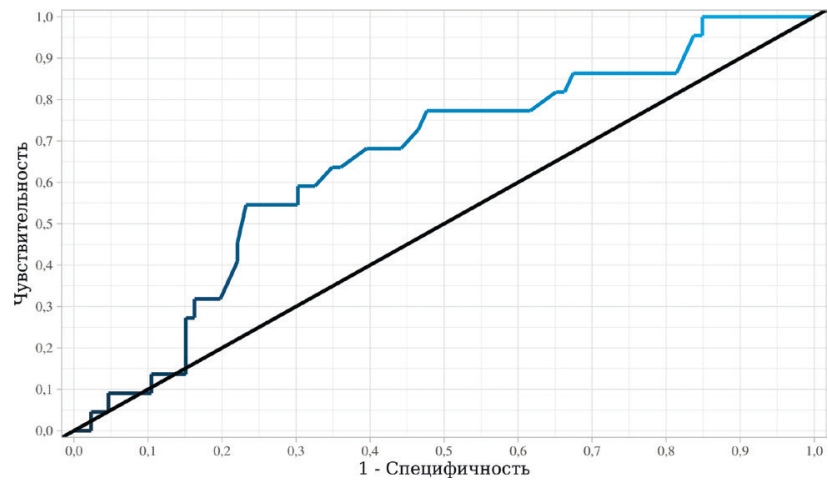

Puc. 1. ROC-кривая, характеризующая зависимость вероятности наличия фибрилляции предсердий от уровня креатинина.
Шансы выявления ФП в группе с содержанием креатинина 103,4 мкмоль/л и более были выше в 3,070 раза, по сравнению с группой с уровнем креатинина менее 103,4 мкмоль/л, различия шансов были статистически значимыми (95\% ДИ: 1,152-8,184) (p=0,021).

\section{Анализ групп с различным бременем ФП}

Среди пациентов с выявленной ФП преобладали пациенты с бременем ФП за сутки $0,1 \%$ и менее - 10 $(45,5 \%)$ пациентов, бремя ФП 0,3-12,2\% наблюдалось у 7 (31,8\%) пациентов и бремя ФП 100\% у 5 (22,7\%) пациентов. Нами был выполнен анализ факторов в зависимости от бремени ФП за сутки. При оценке демографических характеристик и риска инсульта по шкале $\mathrm{CHA}_{2} \mathrm{DS}_{2}$-VASc в зависимости от групп бремени ФП за сутки, не удалось выявить статистически значимых различий. Был выполнен анализ диагноза пациента в зависимости от групп бремени ФП за сутки. В результате сравнения групп с различным бременем ФП за сутки по наличию артериальной гипертензии (АГ), а также по степени АГ, были установлены существенные различия $(\mathrm{p}=0,034$ и $\mathrm{p}=0,043$, соответственно). Сопоставление групп по лабораторным показателям выявило ряд закономерностей. При анализе NT-proBNP, D-димера, уровня креатинина в зависимости от групп бремени ФП за сутки, были установлены статистически значимые различия ( $\mathrm{p}=0,037, \mathrm{p}=0,031, \mathrm{p}=0,036$ соответственно) между группами бремени ФП 0,1\%; 0,3-12,2\% и 100\%. Табл. 5 и 6 демонстрируют результаты сопоставления.

Таблица 4.

Анализ лабораторных и инструментальных данных пациентов в зависимости от наличия ФП

\begin{tabular}{|l|c|c|c|}
\hline Показатель & Отсутствие ФП & Наличие ФП & $\mathrm{p}$ \\
\hline NT-рroВNР, пг/мл & $1882[1166-4432]$ & $1853[1323-4480]$ & 0,912 \\
\hline D- димер, мкг/мл & $0,32[0,24-0,8]$ & $0,68[0,2-1,68]$ & 0,324 \\
\hline Креатинин, мкмоль/л & $86,2[70,3-100,8]$ & $103,4[87,7-112,8]$ & $0,027 *$ \\
\hline СКФ, мл/мин/1,73м² & $79[65-90]$ & $66[59-88]$ & 0,186 \\
\hline Глюкоза, мг/дл & $102[92-119]$ & $100,25[92,9-116]$ & 0,991 \\
\hline Холестерин, ммоль/л & $4,77 \pm 1,34(4,49-5,05)$ & $5,14 \pm 1,2(4,6-5,69)$ & 0,243 \\
\hline Фибриноген, г/л & $3,3[2,7-3,9]$ & $3,31[2,6-4,08]$ & 0,978 \\
\hline Стимуляция ПЖ, \% & $1[0,0-5,5]$ & $11[9,0-53,5]$ & $0,043 *$ \\
\hline
\end{tabular}

Примечание: здесь и далее СКФ - скорость клубочковой фильтрации, ПЖ правый желудочек, * - достоверность различий между группами.

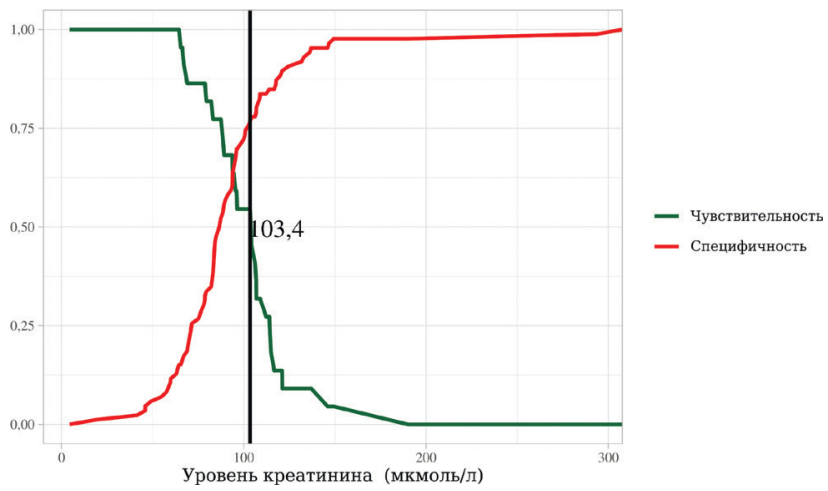

Рис. 2. Анализ соотночения чувствительности и специфичности наличия фибрилляции предсердий в зависимости от уровня креатинина. 


\section{ОБСУЖДЕНИЕ ПОЛУЧЕННЫХ РЕЗУЛЬТАТОВ}

В нашем исследовании были получены следующие результаты: среди пациентов, находящихся на удалённом мониторинге с имплантированными сердечными устройствами, впервые выявленная ФП была зарегистрирована в $19,8 \%$ случаев. Риск инсульта среди этих пациентов был высоким 2 (Me [IQR 1; 3]) балла по шкале $\mathrm{CHA}_{2} \mathrm{DS}_{2}$-VASc у мужчин, и 3 (Me [IQR $2,75 ; 3,75])$ балла у женщин) и требовал рассмотрения вопроса об антикоагулянтной терапии. После классификации случаев ФП в зависимости от бремени ФП за сутки были установлены следующие взаимосвязи: наличие АГ 2 и 3 степени было зафиксировано у всех пациентов в группе с бременем ФП 100\% за сутки. Что статистически значимо отличалось от 2 других групп, где доля пациентов с АГ составляла $30 \%$ в группе с бременем ФП 0,1\% и 42,9\% в группе с бременем ФП 0,3-12,2\%. При анализе лабораторных данных в группах с разным бременем ФП за сутки было установлено, что в группе с бременем ФП 100\% уровни NT-proBNP, D-димера и креатинина были статистически значимо выше, чем в остальных группах. При анализе зависимости уровня креатинина от наличия ФП пороговое значение уровня креатинина в точке cut-off, составило 103,4 мкмоль/л.

В исследовании G.Boriani et al. (2017) доля выявленной ФП у пациентов с имплантированными устройствами была $34,1 \%$. Авторы данной работы также установили, что в группе с ФП возраст пациентов был выше, преобладали пациенты мужского пола, а также реже встречались пациенты с сахарным диабетом. В то время как в нашей работе подобной корреляции

Таблица 5. Анализ клинических данных пациентов в зависимости от бремени ФП за сутки

\begin{tabular}{|c|c|c|c|c|c|}
\hline \multirow{2}{*}{\multicolumn{2}{|c|}{ Показатель }} & \multicolumn{3}{|c|}{ Бремя ФП за сутки } & \multirow{2}{*}{$\mathrm{p}$} \\
\hline & & \multirow{2}{*}{$\frac{0,1 \%}{3(30)}$} & \multirow{2}{*}{$\frac{0,3-12,2 \%}{3(42,86)}$} & \multirow{2}{*}{$\begin{array}{l}100 \% \\
2(40)\end{array}$} & \\
\hline \multirow{4}{*}{ КМП ,n (\%) } & Отсутствие & & & & \multirow{4}{*}{0,702} \\
\hline & ИКМП & $2(20)$ & $3(42,86)$ & $1(20)$ & \\
\hline & ДКМП & $3(30)$ & - & - & \\
\hline & ГКМП & $2(20)$ & $1(14,29)$ & $2(40)$ & \\
\hline \multirow{2}{*}{ ПИМ, n (\%) } & Отсутствие & $7(70)$ & $4(57,14)$ & $3(60)$ & \multirow{2}{*}{0,858} \\
\hline & Наличие & $3(30)$ & $3(42,86)$ & $2(40)$ & \\
\hline \multirow{2}{*}{$\begin{array}{l}\text { Инсульт } \\
\text { в анамнезе, n (\%) }\end{array}$} & Отсутствие & $10(100)$ & $6(85,71)$ & $5(100)$ & \multirow{2}{*}{0,542} \\
\hline & Наличие & - & $1(14,29)$ & - & \\
\hline \multirow{5}{*}{$\Phi К ~ X C H, n(\%)$} & Отсутствие & $3(30)$ & - & - & \multirow{5}{*}{0,5} \\
\hline & ХCH I ФК & $1(10)$ & - & - & \\
\hline & XCH II ФК & $3(30)$ & $3(42,86)$ & $1(20)$ & \\
\hline & XCH III ФК & $3(30)$ & $3(42,86)$ & $3(60)$ & \\
\hline & XCH IV ФК & - & $1(14,29)$ & $1(20)$ & \\
\hline \multirow{2}{*}{$\begin{array}{l}\text { Наличие ХCH, } \\
\text { n }(\%)\end{array}$} & Отсутствие & $3(30)$ & - & - & \multirow{2}{*}{0,279} \\
\hline & Наличие & $7(70)$ & $7(100)$ & $5(100)$ & \\
\hline \multirow{2}{*}{ СД, n (\%) } & Отсутствие & $8(80)$ & $6(85,71)$ & $4(80)$ & \multirow{2}{*}{1} \\
\hline & Наличие & $2(20)$ & $1(14,29)$ & $1(20)$ & \\
\hline \multirow{2}{*}{ ДСУ, n (\%) } & Отсутствие & $10(100)$ & $7(100)$ & $4(80)$ & \multirow{2}{*}{0,225} \\
\hline & Наличие & - & - & $1(20)$ & \\
\hline \multirow{3}{*}{$\begin{array}{l}\text { Степень АГ, } \\
\mathrm{n}(\%)\end{array}$} & Отсутствие & $7(70)$ & $4(57,14)$ & - & \multirow{3}{*}{$0,043 *$} \\
\hline & АГ II ст. & $1(10)$ & - & $2(40)$ & \\
\hline & АГ III ст. & $2(20)$ & $3(42,86)$ & $3(60)$ & \\
\hline \multirow{2}{*}{$\begin{array}{l}\text { Наличие АГ, } \\
\text { n (\%) }\end{array}$} & Отсутствие & $7(70)$ & $4(57,14)$ & - & \multirow{2}{*}{$0,034 *$} \\
\hline & Наличие & $3(30)$ & $3(42,86)$ & $5(100)$ & \\
\hline \multirow{2}{*}{ ПБЛНПГ, n (\%) } & Отсутствие & $6(60)$ & $3(42,86)$ & $3(60)$ & \multirow{2}{*}{0,868} \\
\hline & Наличие & $4(40)$ & $4(57,14)$ & $2(40)$ & \\
\hline \multirow{2}{*}{ ППС, n (\%) } & Отсутствие & $9(90)$ & $7(100)$ & $4(80)$ & \multirow{2}{*}{0,698} \\
\hline & Наличие & $1(10)$ & - & $1(20)$ & \\
\hline \multirow{2}{*}{ ЖТ, n (\%) } & Отсутствие & $9(90)$ & $6(85,71)$ & $5(100)$ & \multirow{2}{*}{1} \\
\hline & Наличие & $1(10)$ & $1(14,29)$ & - & \\
\hline
\end{tabular}

выявлено не было, что возможно связано с меньшим количеством пациентов. В этой работе исследователи также рассмотрели предикторы перехода ФП в более продолжительную форму. Аналогично нашему исследованию в данной работе была установлена связь артериальной гипертензии с бременем ФП. В нашем исследовании прослеживалась статистически значимая связь наличия и степени артериальной гипертензии с более высоким бременем ФП, а в вышеупомянутом исследовании коллег артериальная гипертензия являлась независимым предиктором перехода ФП в более продолжительную форму [8]. В исследовании, которое включало еще большее количество пациентов, чем предыдущее (SOS-AF), a именно более 10000 пациентов, доля выявленной ФП составила $43 \%$ и бремя ФП равное 1 час, было ассоциировано с более высоким риском развития ишемического инсульта [9]. Доля субклинической ФП у пациентов из исследования ASSERT составила $18,8 \%$, что ненамного меньше, чем в настоящем исследовании. Однако, следует отметить, что в этом исследовании учитывались лишь эпизоды, длящиеся более 6 минут. Исследователи в данной работе выбрали немного другой подход к учету бремени ФП, а именно, они учитывали самый длинный непрерывный эпизод ФП. Анализируя связь длительности одиночного эпизода ФП и риск инсульта, учёные пришли к выводу, что у пациентов с продолжительностью ФП 
более 24 часов имеется высокий риск ишемического инсульта и системной эмболии [10].

Высокий уровень мозгового натрийуретического пептида (NT-proBNP) говорит о наличии и тяжести хронической сердечной недостаточности (ХCH). Повышенный уровень этого пептида в группе пациентов с бременем ФП 100\% в нашем исследовании возможно, является следствием большего количества пациентов с XСН ФК III в этой группе пациентов (60\% vs 30\% и 42,9\% в двух других группах). По данным исследований частота встречаемости ФП среди пациентов с ХСН составляет 34\%, а среди пациентов с ХСН частота ФП равна 42\%. Такую тесную взаимосвязь этих двух состояний можно частично объяснить общими факторами риска в виде АГ, ишемической болезни сердца, кардиомиопатии, сахарного диабета и других [13-15].

Хроническая болезнь почек (ХБП) является частым коморбидным состоянием при ХCH и расценивается как фактор повышающий смертность и риск осложнений. Снижение систолической функции левого желудочка приводит к ухудшению функции почек что, в свою очередь, вызывает прогрессирование ХСН $[16,17]$. Повышенный уровень креатинина у пациентов с высоким бременем ФП в нашем исследовании можно расценить как показатель снижения функционального состояния почек. По данным мета-анализа с числом обследуемых более 80000 пациентов с ХCH, ХБП встречалась у 63\% больных. Около трети этих пациентов страдали умеренной или тяжелой ХБП. Исследователи в данной работе установили также связь смертности в течение года и ХБП. Смертность была выше у пациентов с выраженной ХБП [18].

Исследование Т.Horio и соавторов показало, что осложнение ХБП, особенно прогрессирующая почечная дисфункция, была сильным предиктором впервые выявленной ФП у пациентов с гипертонией. Регрессионный анализ Кокса в данном исследовании продемонстрировал, что возраст, курение, размер левого предсердия, индекс массы левого желудочка и наличие ХБП были значимо связаны с возникновением ФП. Среди этих возможных предикторов ХБП (ОР $2,18, \mathrm{P}=0,009)$ была независимым предиктором развития ФП [19]. В мета-анализе трех когорт сниженная скорость клубочковой фильтрации и повышенное соотношение альбумина к креатинину в моче были значительно связаны с повышенным риском развития фибрилляции предсердий [20].
В проспективном когортном обсервационном исследовании, включавшем 235818 пациентов, проведенном в рамках добровольной ежегодной программы медицинского осмотра в Японии была изучена связь нарушения функции почек при поступлении с последующим возникновением ФП и связь существующей ФП с развитием почечной недоста-

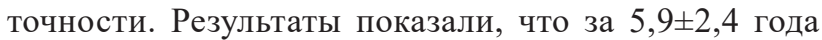
ФП развилась у 2947 пациентов (1,3\%). Исходный уровень креатинина сыворотки и скорость клубочковой фильтрации были связаны с риском развития ФП. Фибрилляция предсердий при поступлении была связана с развитием ХБП (ОР [95\% ДИ], 1,77 $[1,50-2,10])$ и протеинурии (ОР [95\% ДИ], 2,20 [1,92$2,52])$. Связь наблюдалась у пациентов без терапии АГ или сахарного диабета. Иными словами, наблюдалось взаимное влияние этих двух патологий друг на друга. ХБП увеличивала риск возникновения ФП, а ФП увеличивала риск развития ХБП [21].

Повышенный уровень D-димера в нашем исследовании положительно коррелировал с высоким бременем ФП. D-димер является продуктом распада фибрина и определяется в крови при помощи лабораторного теста. Существует ряд работ подтверждающих роль D-димера в предсказании тромбоэмболических осложнений у пациентов с ФП [22, 23]. В исследовании A. Zorlu et al. (2012) была установлена роль D-димера как предиктора развития ФП у пациентов с XCH $(\mathrm{p}=0,006)$. Более того, повышенный уровень D -димера в этом исследовании был ассоциирован с повышенной смертностью от кардиоваскулярных событий независимо от наличия ФП у пациентов с ХСН [24].

Появляется все больше свидетельств того, что стимуляция правого желудочка увеличивает риск ФП в долгосрочной перспективе, что было продемонстрировано учеными в исследовании MOST. В 2003 г. M.Sweeney и соавторы опубликовали ретроспективный анализ 1339 пациентов с нормальной продолжительностью QRS из исследования MOST. Медиана желудочковой стимуляции (\%) была выше при двухкамерной стимуляции (DDDR) по сравнению с однокамерной желудочковой стимуляцией (VVIR) (90\% vs. 58\%, $\mathrm{p}=0,001)$. Риск ФП увеличивался линейно с увеличением процента желудочковой стимуляции от $0 \%$ до $85 \%$. Риск ФП увеличивается на 1\% и 0,7\% на каждый 1\% увеличения стимуляции желудочков до $80 \%$ в DDDR и VVIR, соответственно [25].

Таблица 6.

Анализ лабораторных и инструментальных данных пациентов в зависимости от бремени ФП за сутки

\begin{tabular}{|l|l|l|l|l|}
\hline \multirow{2}{*}{ Показатель } & \multicolumn{3}{|c|}{ Бремя ФП за сутки } & \multirow{2}{*}{ p } \\
\cline { 2 - 5 } & \multicolumn{1}{|c|}{$0,1 \%$} & \multicolumn{1}{|c|}{$0,3-12,2 \%$} & $100 \%$ & \\
\hline NT-ргоВNР, пг/мл & $1781[1067-2696]$ & $1383[1143-1512]$ & $6387[4822-7663]$ & $0,037^{*}$ \\
\hline D-димер, мкг/мл & $0,53[0,24-0,74]$ & $0,16[0,14-0,32]$ & $1,69[1,61-2,01]$ & $0,031^{*}$ \\
\hline Креатинин, мкмоль/л & $93 \pm 18(80-107)$ & $95 \pm 18(77-114)$ & $121 \pm 22(91-151)$ & $0,036^{*}$ \\
\hline Глюкоза, мг/дл & $104[92,7-118,47]$ & $98[94-103]$ & $106[99-151]$ & 0,666 \\
\hline Холестерин, ммоль/л & $5,06 \pm 1,05(4,27-5,86)$ & $5,54 \pm 1,62(3,93-7,16)$ & $4,73 \pm 0,83(3,58-5,88)$ & 0,519 \\
\hline Фибриноген, г/л & $2,98[2,6-3,88]$ & $2,84[2,54-3,82]$ & $4,5[3,31-5,2]$ & 0,505 \\
\hline
\end{tabular}


Стимуляция правого желудочка отрицательно влияет на структуру и функцию левого предсердия, что может вызвать ФП. Сравнивается риск возникновения персистирующей и постоянной ФП во время длительной стимуляции пучка Гиса, перегородки правого желудочка и верхушки правого желудочка у пациентов с полной и прогрессирующей атриовентрикулярной блокадой. Риск возникновения персистирующей / постоянной ФП был значительно ниже у пациентов, которым проводилась стимуляция пучка Гиса, по сравнению с пациентами, которым проводилась стимуляция верхушки правого желудочка $(\mathrm{OP}=0,28,95 \%$ ДИ 0,160,48, $\mathrm{p}=0,0001)$. Группы стимуляции перегородки правого желудочка и верхушки правого желудочка показали одинаковый риск ФП (ОР 1,04, 95\% ДИ 0,66-1,64, $\mathrm{p}=0,856)[26]$.

Ограничением настоящего исследования следует считать невозможность проследить клинические исхо- ды, в частности тромбоэмболические осложнения ФП за период наблюдения.

\section{ЗАКЛЮЧЕНИЕ}

Мы пришли к выводу, что впервые выявленная ФП встречается у $1 / 5$ пациентов с имплантированными сердечными устройствами. Все пациенты с ФП характеризовались высоким риском инсульта и большинство пациентов являлись потенциальными кандидатами на антикоагулянтную терапию. Частота стимуляции правого желудочка у пациентов с ИКд была выше в группе пациентов с ФП. Суточное бремя ФП положительно коррелирует с наличием и степенью АГ, а также с маркерами нарушения функции почек, хронической сердечной недостаточности и тромбоза. Повышенный уровень креатинина является предиктором развития ФП у пациентов с имплантированными сердечными устройствами.

\section{ЛИТЕРАТУРА}

1. January CT, Wann LS, Calkins H, et al. 2019 AHA/ ACC/HRS Focused Update of the 2014 AHA/ACC/HRS Guideline for the Management of Patients With Atrial Fibrillation. Circulation. 2019;139: 2502-2512. https://doi. org/10.1161/CIRCULATIONAHA.118.038988.

2. Kirchhof P, Benussi S, Kotecha D, et al. Authors/Task Force Members, Document Reviewers:. 2016 ESC Guidelines for the Management of Atrial Fibrillation Developed in Collaboration with EACTS: The Task Force for the Management of Atrial Fibrillation of the European Society of Cardiology (ESC) developed with the special contribution of the European Heart Rhythm Association (EHRA) of the ESCEndorsed by the European Stroke Organisation (ESO). Eur Heart J. 2016;38: 2893-2962.

3. Dilaveris PE, Kennedy HL. Silent atrial fibrillation: epidemiology, diagnosis, and clinical impact. Clin Cardiol. 2017;40(6): 413-418. https://doi.org/10.1002/ clc.22667. Epub 2017 Mar 8. PMID: 28273368; PMCID: PMC6490532.

4. Hindricks G, Potpara T, Dagres N, et al. ESC Scientific Document Group. 2020 ESC Guidelines for the diagnosis and management of atrial fibrillation developed in collaboration with the European Association for Cardio-Thoracic Surgery (EACTS): The Task Force for the diagnosis and management of atrial fibrillation of the European Society of Cardiology (ESC) Developed with the special contribution of the European Heart Rhythm Association (EHRA) of the ESC. Eur Heart J. 2021;42(5): 373-498 https://doi. org/10.1093/eurheartj/ehaa612.

5. Al-Jefairi N, Burri H. Relevance of guideline-based ICD indications to clinical practice. Indian Heart J. 2014;66: S82-S87. https://doi.org/10.1016/j.ihj.2013.11.006.

6. Lawin D, Stellbrink C. Change in indication for cardiac resynchronization therapy? Eur J Cardiothorac Surg. 2019;55: i11-i16. https://doi.org/10.1093/ejcts/ezy488.

7. Zacà V, Marcucci R, Parodi G, et al. Management of antithrombotic therapy in patients undergoing electrophysiological device surgery. Europace. 2015;17(6): 840-54. https://doi.org/10.1093/europace/euu357.

8. Boriani G, Glotzer TV, Ziegler PD, et al. Detection of new atrial fibrillation in patients with cardiac implanted electronic devices and factors associated with transition to higher device-detected atrial fibrillation burden. Heart Rhythm. 2018;15(3): 376-383. https://doi.org/10.1016/j. hrthm.2017.11.007.

9. Boriani G, Glotzer TV, Santini M, et al. Device-detected atrial fibrillation and risk for stroke: an analysis of $>10,000$ patients from the SOS AF project (Stroke preventiOn Strategies based on Atrial Fibrillation information from implanted devices). Eur Heart J. 2014;35(8): 508-16. https://doi.org/10.1093/eurheartj/eht491.

10. Van Gelder IC, Healey JS, Crijns HJGM, et al. Duration of device-detected subclinical atrial fibrillation and occurrence of stroke in ASSERT. Eur Heart J. 2017;38(17): 1339-1344. https://doi.org/10.1093/eurheartj/ehx042.

11. Deharo JC, Djiane P. Home Monitoring: what can we expect in the future? Clin Res Cardiol. 2006;95: 36-9. https://doi.org/10.1007/s00392-006-1307-5.

12. Oliveira M, Fernandes M, Primo J, et al. Remote versus face-to-face monitoring for implantable cardiac devices: rationale and design of the PORTLink (PORTuguese Research on Telemonitoring with CareLink) trial. Rev Port Cardiol. 2013;32(12): 957-64. https://doi.org/10.1016/j. repc.2013.06.009.

13. Nieuwlaat R, Capucci A, Camm AJ, et al. European Heart Survey Investigators. Atrial fibrillation management: a prospective survey in ESC member countries: the Euro Heart Survey on Atrial Fibrillation. Eur Heart J. 2005;26(22): 2422-34. https://doi.org/10.1093/eurheartj/ ehi505.

14. Cleland JG, Swedberg K, Follath F, et al. Study Group on Diagnosis of the Working Group on Heart Failure of the European Society of Cardiology. The EuroHeart Failure survey programme - a survey on the quality of care among patients with heart failure in Europe. Part 1: patient characteristics and diagnosis. Eur Heart J. 2003;24(5): 442-63. https://doi.org/10.1016/s0195$668 \times(02) 00823-0$.

15. Khan MA, Ahmed F, Neyses L, et al. Atrial fibrillation in heart failure: The sword of Damocles revisited. World J Cardiol. 2013;5(7): 215-27. https://doi.org/10.4330/wjc. v5.i7.215. 
16. Кутырина ИМ, Швецов МЮ. Возможности лечения почечной артериальной гипертонии. Качество жизни. Медиичина. 2006;4(15): 78-89. [Kutyrina IM, Shveczov MYu. Vozmozhnosti lecheniya pochechnoj arterial'noj gipertonii. Kachestvo zhizni. Mediczina. 2006;4(15): 7889 (In Russ.)].

17. Моисеев ВС, Кобалава ЖД. Кардиоренальный синдром (почечный фактор и повышение риска сердечно-сосудистых заболеваний). Клин фармак тер. 2002;11 (3): 16-18. [Moiseev VS, Kobalava ZhD. Kardiorenal`ny`j sindrom (pochechny`j faktor i povy`shenie riska serdechno-sosudisty`kh zabolevanij). Klin farmak ter. 2002;11(3): 16-18 (In Russ.)].

18. Smith GL, Lichtman JH, Bracken MB, et al. Renal impairment and outcomes in heart failure: systematic review and meta-analysis. J Am Coll Cardiol. 2006;47(10): $1987-$ 96. https://doi.org/10.1016/j.jacc.2005.11.084.

19. Horio T, Iwashima Y, Kamide K, et al. Chronic kidney disease as an independent risk factor for new-onset atrial fibrillation in hypertensive patients. Journal of Hypertension. 2010;28(8): 1738-1744. https://doi.org/10.1097/ hjh.0b013e32833a7dfe.

20. Bansal N, Zelnick LR, Alonso A, et al. eGFR and Albuminuria in Relation to Risk of Incident Atrial Fibrillation: A Meta-Analysis of the Jackson Heart Study, the Multi-Ethnic Study of Atherosclerosis, and the Cardiovascular Health Study. Clin J Am Soc Nephrol. 2017;12(9): 1386-1398. https://doi.org/10.2215/CJN.01860217.
21. Watanab H, Watanabe T, Sasaki S, et al. Close bidirectional relationship between chronic kidney disease and atrial fibrillation: The Niigata preventive medicine study. American Heart Journal. 2009;158(4): 629-636. https:// doi.org/10.1016/j.ahj.2009.06.031.

22. Yashiro Y, Arimoto T, Hashimoto N, et al. Predictors of left atrial coagulation activity among paroxysmal atrial fibrillation patients. Circ J. 2015;79(1): 61-9. https://doi. org/10.1253/circj.CJ-14-0630..

23. Sadanaga T, Sadanaga M, Ogawa S. Evidence that D-dimer levels predict subsequent thromboembolic and cardiovascular events in patients with atrial fibrillation during oral anticoagulant therapy. J Am Coll Cardiol. 2010;55(20): 2225-31. https://doi.org/10.1016/j. jacc.2009.12.049.

24. Zorlu A, Akkaya E, Altay H, et al. The relationship between D-dimer level and the development of atrial fibrillation in patients with systolic heart failure. J Thromb Thrombolysis. 2012;33(4): 343-8. https://doi.org/10.1007/ s11239-011-0656-8. PMID: 22139027.

25. Sweeney MO, Hellkamp AS, Ellenbogen KA et al. Adverse effect of ventricular pacing on heart failure and atrial fibrillation among patients with normal baseline QRS duration in a clinical trial of pacemaker therapy for sinus node dysfunction. Circulation. 2003;107: 2932 -7.

26. Pastore G, Zanon F, Baracca E et al. The risk of atrial fibrillation during right ventricular pacing. Europace. 2016;18(3): 353-8. https://doi.org/10.1093/europace/euv268. 